\title{
On the analysis and design of a fully compliant large stroke slider-crank (rocker) mechanism
}

\author{
Çağıl Merve Tanık ${ }^{1}$, Engin Tanık ${ }^{2}$, Yiğit Yazıcıoğlu ${ }^{1}$, and Volkan Parlaktaş ${ }^{2}$ \\ ${ }^{1}$ Department of Mechanical Engineering, Middle East Technical University, 06800 Ankara, Turkey \\ ${ }^{2}$ Department of Mechanical Engineering, Hacettepe University, 06800 Ankara, Turkey \\ Correspondence: Engin Tanık (etanik@hacettepe.edu.tr)
}

Received: 27 May 2019 - Revised: 1 January 2020 - Accepted: 20 January 2020 - Published: 7 February 2020

\begin{abstract}
In the literature, authors have made contributions in the area of partially compliant slider-crank (rocker) mechanisms possessing rigid joints that may cause backlash inherently. On contrary, fully compliant mechanisms offer no backlash which is a valuable property for the cases where high precision is required. In this paper, we proposed a fully compliant slider-crank mechanism that performs large stroke. Kinematic performance of the mechanism is investigated analytically. Dimensions of the mechanism are optimized to obtain maximum translational output, while keeping deflections of flexible hinges equal to each other and as small as possible. A design table displaying stroke, axis drift of the output segment, and critical stresses of compliant segments are presented. As an example, a compliant mechanism is designed by using rigid body replacement technique. Then, via nonlinear finite element analysis technique, analytical results are verified. Finally, a prototype is built to compare output stroke and axis drift with analytical approaches. The results of experiments verified that the theoretical approaches are consistent.
\end{abstract}

\section{Introduction}

Compliant mechanisms are flexible mechanisms that transfer some or all of their motion through deformation of elastic segments. They are divided into two main categories; partially or fully compliant mechanism. Partially compliant mechanisms have at least one traditional (rigid) joint that may cause backlash inherently. By definition, a fully compliant mechanism does not possess a conventional rigid joint. Thus, fully compliant mechanisms obtain all their motion from deflection of compliant segments (Howell, 2001). This property is advantageous for the cases where precision is crucial. Compliant mechanisms have further advantages such as low cost, reduced number of parts, no need for lubrication, less wear and noise. Additionally, stored elastic energy due to deformation of compliant members brings mechanism to its original position. Pseudo-rigid-body model (PRBM) is used to simplify analysis of systems that undergo large, nonlinear deflections (Howell and Midha, 1996). Yu et al. (2016) proposed a new three degree-of-freedom (DOF) model based on PRBM for large deflection beams. In Liu and Yan's (2017) study modified pseudo-rigid- body modelling approach for compliant mechanisms with fixed guided beam flexurals was examined.

Rigid joints allow specific DOF between connected parts. However, rigid joints suffer from backlash and wear because of clearance between mating parts. Trease et al. (2005) proposed a revolute and translational compliant joint. Another novel three dimensional compliant translational joint with large stiffness ratio and small axis drift was presented (Yang et al., 2016).

Four link mechanisms (e.g. four-bar and slider-crank) have significant importance in the industry. Slider-crank mechanisms have numerous applications especially when kinematic inversions are considered. Researches on compliant slider-crank (rocker) mechanisms are limited in the literature. Hao et al. (2018) proposed a multi-mode compliant gripper. There is a fully compliant slider-crank mechanism available in their gripper. In Parlaktaş and Tanık's (2014) study the "single piece" compliant spatial slider-crank mechanism was introduced. In this study, deflections of the multiple axis flexural hinges were determined separately as bend- 
ing and twist. Erkaya et al. (2016) investigated dynamics of the partially compliant slider-crank mechanism. In Alqasimi et al.'s (2016) study, a new model for a bistable compliant mechanism that is based on crank-slider mechanism was presented. In this study the slider is rigid thus the mechanism is partially compliant. In Pardeshi et al.'s (2017) study, monolithic compliant slider-crank mechanism with a rigid slider for motion amplification was proposed. Dao and Huang (2014) proposed an optimal design of a partially compliant slider-crank mechanism with circular cross-section flexure hinges.

Furthermore, there are some studies based on compliant linear-motion mechanisms which can not be categorized as slider-crank mechanism: Pavlović and Pavlović (2009) introduced compliant parallel-guiding mechanism's design procedure. A new compliant mechanism which is capable to realize axial translation of the link was presented. An inherent parasitic motion of the compliant parallel four-bar mechanism was compensated by exploiting center shift of a generalized cross-spring pivot in Hongzhe et al.'s (2012) study. Zhao, et al. (2017) designed a stiffness-adjustable compliant linear-motion mechanism.

Partially compliant mechanisms possess rigid prismatic joints (slider) in their structure. Prismatic joints inherently have disadvantages due to backlash and friction problems. An approach for optimum link proportions of the rigid body equivalent is proposed. The optimization objective is to maximize the translational motion of the slider equivalent while minimizing the relative link rotations. Minimization of link rotations is essential to keep maximum stresses in an acceptable range. Input-output motion relationships of the mechanism are determined. Resultant stresses at flexural hinges are obtained analytically. A design table is prepared for generalization of the dimensions that will be beneficial for other researchers. As a design example, an optimum mechanism is analyzed via finite element analysis (FEA) method and analytical results are verified. A prototype is manufactured and experiments are conducted. Also, it is ensured that the results of experiments are consistent with the theoretical approaches.

Note that, for the cases where crank of a slider-crank mechanism does not fully rotate, the mechanism may be called as slider-rocker mechanism. However, this makes an ambiguity for the identity of the mechanism. In this study, we prefer to name the mechanism as slider-crank disregarding the link proportions.

\section{The proposed fully compliant slider-crank mechanism}

In Fig. 1a, a partially compliant slider-crank mechanism that possesses a prismatic joint between the slider and ground is presented. In our design, we replaced the prismatic joint by two identical and parallel compliant segments and a rigid segment as shown in Fig. 1b. By this way, if properly designed, translational motion for the output which acts as a slider can be achieved. Alternatively, compliant version of Roberts or Watt (Shigley and Uicker, 1980) type four bar mechanisms can be implemented for slider replacement. Specific points on coupler link of these mechanisms trace approximate straight lines. However, their coupler link performs rotation as well as translation. In our case, the output link performs no rotation but only translation (curvilinear translation). In the literature, paired double parallelogram mechanisms are used as sliders (Trease et al., 2005; Li et al., 2018). The advantage of this structure is straight line motion with no rotation. However, there is a big major disadvantage; if PRBM of this type is constructed, it can be calculated that DOF $<0$. This case yields a locking mechanism. However, compliant version of this mechanism moves with axial deformation of compliant segments as well as bending. This property increases the stresses, therefore decreases stroke of the mechanism dramatically. Our major concern in this study is to increase the stroke as high as possible while keeping the stresses in an acceptable range.

In Fig. 2a, PRBM of the fully compliant slider-crank mechanism is displayed. This PRBM is essentially a six-link mechanism, where two of the links are connected to a parallelogram four-bar mechanism. The coupler link of this parallelogram four-bar acts as a slider, if the fixed guided segments 1 and 2 are long enough and rigid segment 4 stays parallel to its initial position (Fig. 2b). These fixed guided segments must be identical, initially straight, and parallel to each other. By this way, we obtain a compliant parallelguiding mechanism (Howell, 2001) where rigid segment 4 performs curvilinear translation. In the case of an extreme vertical load, fixed guided segments may buckle thus, rigid segment 4 becomes nonparallel to its initial position.

\section{Kinematic analysis of the cascade parallelogram four-bar mechanism}

Initially, kinematic analysis of the PRBM which is essentially a rigid cascade parallelogram four-bar mechanisms is performed. The PRBM (Fig. 3) is formed as follows: input (link 2) and connecting rod (link 3 ) of the mechanism are combined with a parallelogram four-bar mechanism whose coupler link (link 4) acts as a slider of the fully compliant slider-crank mechanism (Fig. 2b).

Kutzbach criterion (Shigley and Uicker, 1980) for DOF of a planar mechanism is $N=3(l-1)-2 j_{1}-j_{2}$ where $j_{1}$ refers to the number of single DOF joints and $j_{2}$ refers to the number of two DOF joints. The PRBM has six links and seven revolute joints. Thus, according to Kutzbach criterion, DOF of the mechanism is calculated as $N=1$.

Referring to Fig. 3, the revolute joints at $A_{0}, C_{0}$, and $D_{0}$ are pivoted to the ground. Length of $r_{1}$ is $A_{0} C_{0}$, length of link 2 is $r_{2}=A_{0} A$, length of link 3 is $r_{3}=A B$. Link 4 that 


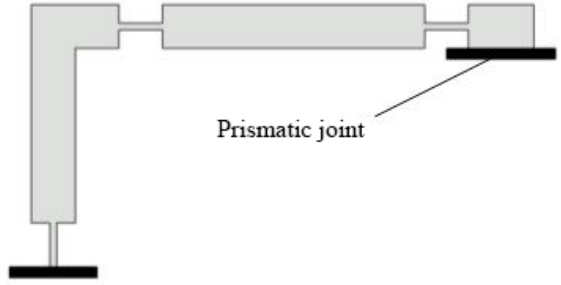

(a)

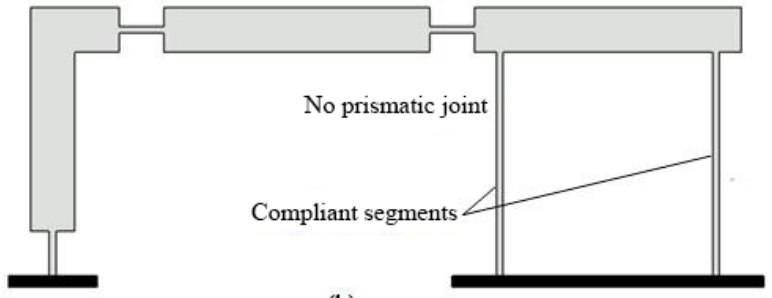

(b)

Figure 1. (a) Partially compliant slider-crank with prismatic joint and (b) proposed fully compliant slider-crank.

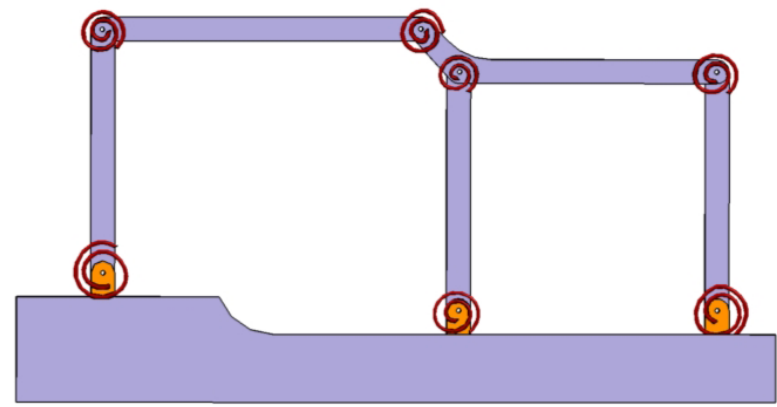

(a)

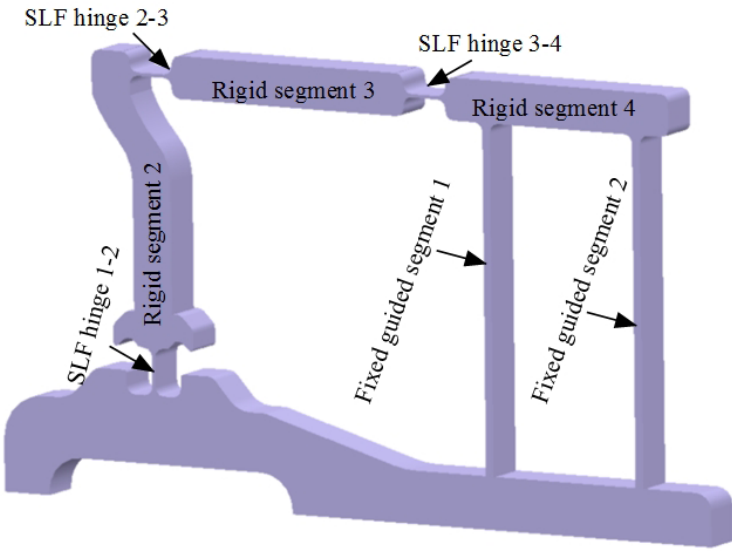

(b)

Figure 2. (a) PRBM (b) isometric view of the mechanism whose slider is composed of fixed guided compliant segments.

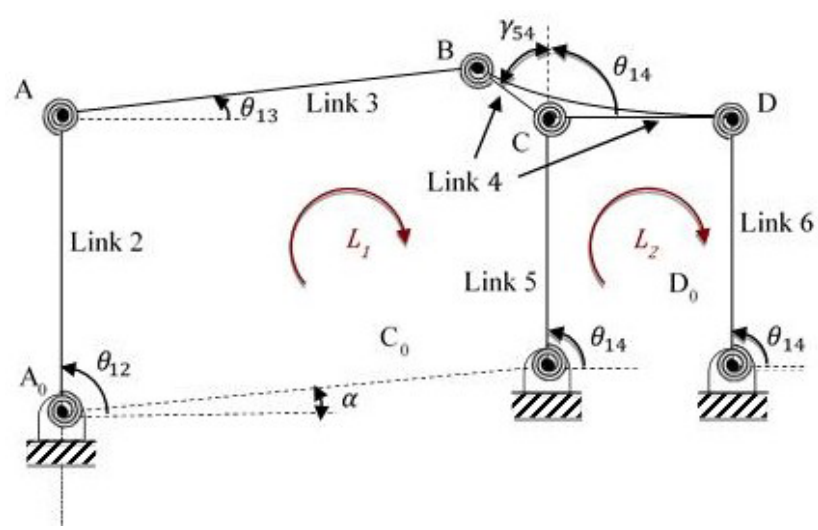

Figure 3. Structure parameters and variables for the kinematic analysis.

is formed by BCD is single piece and $r_{8}=B C, r_{4}=C D$, length of links 5 and 6 is $r_{5}=C_{0} C=r_{6}=D_{0} D$. The horizontal distance between the revolute joints of the parallelogram four-bar is $r_{10}=C_{0} D_{0}=r_{4} . \theta_{i j}$ are position variables measured counter-clockwise (in the right handed sense) from the horizontal axis. $\alpha$ is a constant angle between the horizontal axis and $A_{0} C_{0} \cdot \gamma_{54}$ is a variable angle between links 4 and 5 measured counter-clockwise from the vertical axis. Note that, $\gamma_{54}+\theta_{14}$ is a constant angle.

The main objective of the position analysis is to derive closed form equations according to the position variables as a function of input angle $\theta_{12}$ and the structure parameters. There are two independent loops $L_{1}$ and $L_{2}$ as shown in Fig. 3. The relationship between the position variables, the structure parameters, and the input angle is obtained in Eq. (1) by substituting trigonometric identities into the loop closure equations.

$$
\begin{aligned}
r_{3}^{2} & =r_{1}^{2}+r_{2}^{2}+r_{5}^{2}+r_{8}^{2}-2 r_{1} r_{2} \cos \left(\theta_{12}-\alpha\right) \\
& +2 r_{1} r_{5} \cos \left(\theta_{14}-\alpha\right)+2 r_{1} r_{8} \cos \left(\theta_{14}+\gamma_{54}-\alpha\right) \\
& +2 r_{2} r_{5} \cos \left(\theta_{14}-\theta_{12}\right)-2 r_{2} r_{8} \cos \left(\theta_{14}+\gamma_{54}-\theta_{12}\right) \\
& +2 r_{5} r_{8}
\end{aligned}
$$

However, obtaining a closed form relationship between $\theta_{12}$ and $\theta_{14}$ is very hard if not impossible. In the next section, we proposed an alternative method to obtain a closed form solution.

\section{A novel kinematic analysis approach for the PRBM}

It is clear that link 4 performs curvilinear translation motion due to the parallelogram structure of the four-bar mech- 


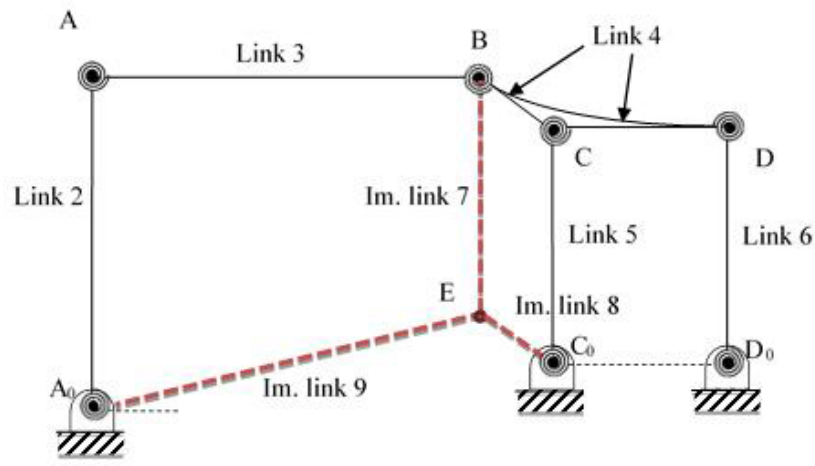

Figure 4. PRBM with an imaginary parallelogram four-bar mechanism.

anism. Thus, any point on the coupler link moves on congruent curves. Points $C$ and $D$ performs fixed axis rotation about $C_{0}$ and $D_{0}$ respectively with a radius of curvature of $r_{5}$. This brings us a very useful property for the analysis of the mechanism described as follows: let an imaginary link 7 is drawn parallel to link 5 between points $B E$ ( $E$ is pivoted to the ground with a revolute joint) as shown in Fig. 4 . The length of imaginary link $7 ; r_{7}$ is equal to $r_{5}$ that is also equal to the radius of curvature. Furthermore, an imaginary link 9 is formed between points $A_{0} E$ with length of $r_{9}$. Finally, an imaginary link 8 is drawn between points $C_{0} E$ with length of $r_{8}$. Thus, an additional imaginary parallelogram four-bar mechanism is formed consisting links $4,5,7$, and 8 . Link 8 is parallel to $C B$ and $C B=r_{8}$. Referring to Fig. $3, \delta$ and $\chi$ are constant angles between the horizontal axis and $A_{0} E$, $C_{0} E$ respectively. $\mu_{73}$ can be called as the transmission angle between links 3 and 7. By this way, the new PRBM transforms to the mechanism in Fig. 4. This mechanism can be analyzed as two cascade four-bar mechanisms.

Initially, kinematic analysis of the first four-bar mechanism in Fig. 4, composed of links 9, 2, 3, and 7 is performed. $\theta_{14}$ is determined as a function of $\theta_{12}$ that is the input of the second four-bar mechanism. Next, kinematic analysis of the second mechanism (links 4, 5, 7, and 8) is performed. Now, all of the necessary position variables of the complete mechanism are determined in another way. Because, once the angle of link 7 w.r.t ground is found, the angles of the links 5 and 6 will be the same due to two coherent parallelograms; $B E C_{0} C$ and $C_{0} C D D_{0}$.

In order to obtain relationship between input $\theta_{12}$ and position variable $\theta_{14}$, the loop closure equation of the first fourbar mechanism can be written as:

$r_{2} e^{i \theta_{12}}+r_{3} e^{i \theta_{13}}=r_{9} e^{i \delta}+r_{7} e^{i \theta_{14}}$.

Multiplying Eq. (2) by its complex conjugate, $\theta_{14}$ can be determined as a function of $\theta_{12}$.

$\theta_{14}=2 \arctan \left(\left(-B \mp \sqrt{B^{2}-4 A C}\right) / 2 A\right)$

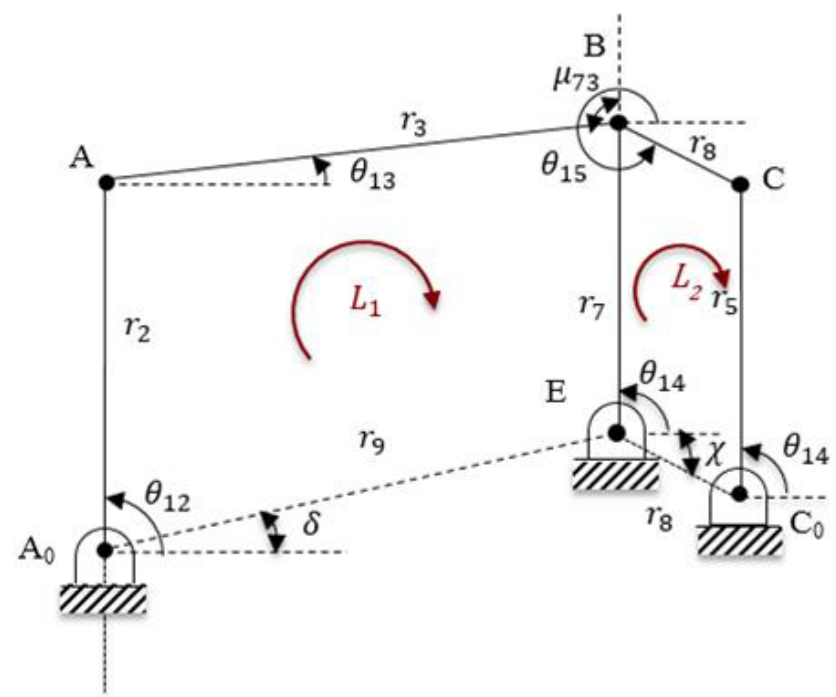

Figure 5. Structure parameters and variables for the cascade fourbar mechanism.

where:

$A=\cos \theta_{12}\left(1-K_{2}\right)+K_{3}-K_{1}$

$B=-2 \sin \theta_{12}$

$C=\cos \theta_{12}\left(1+K_{2}\right)+K_{3}+K_{1}$

$K_{1}=r_{9} / r_{2}$

$K_{2}=r_{9} / r_{7}$

$K_{3}=\left(r_{9}^{2}+r_{2}^{2}-r_{3}^{2}+r_{7}^{2}\right) / 2 r_{2} r_{7}$.

The coupler angle $\left(\theta_{13}\right)$ of the first four-bar mechanism can be determined as

$\theta_{13}=\arg \left[r_{7} e^{i\left(\theta_{14}+\delta\right)}+r_{9}-r_{2} e^{i\left(\theta_{12}+\delta\right)}\right]-\delta$.

Considering the parallelogram shown in Fig. 5, $\theta_{15}$ can be determined as

$\theta_{15}=2 \pi-\chi$.

Referring Fig. 3 input, output, and coupler link angles of the parallelogram mechanism are equal to each other $\left(\theta_{14}\right)$. Therefore, after the kinematic analysis of the cascade fourbar mechanism, slider equivalent part of the mechanism can be readily analyzed.

During rigid body replacement synthesis, if the undeflected position of the compliant segment between rigid segments 3 and 4 is set to $180^{\circ}$, the deflection of this compliant segment will be approximately same in both directions. In addition, $\mu_{73}$ is transmission angle of the PRBM, since the mechanism is equivalent to a slider-crank mechanism. If we define $\mu_{73}=90^{\circ}$ where the transmission angle is optimum, motion quality of the synthesized mechanism will be better. Note that for a compliant mechanism, compliance of flexural hinges may cause differences in transmission characteristics when compared to its rigid body counterpart (Tanık, 


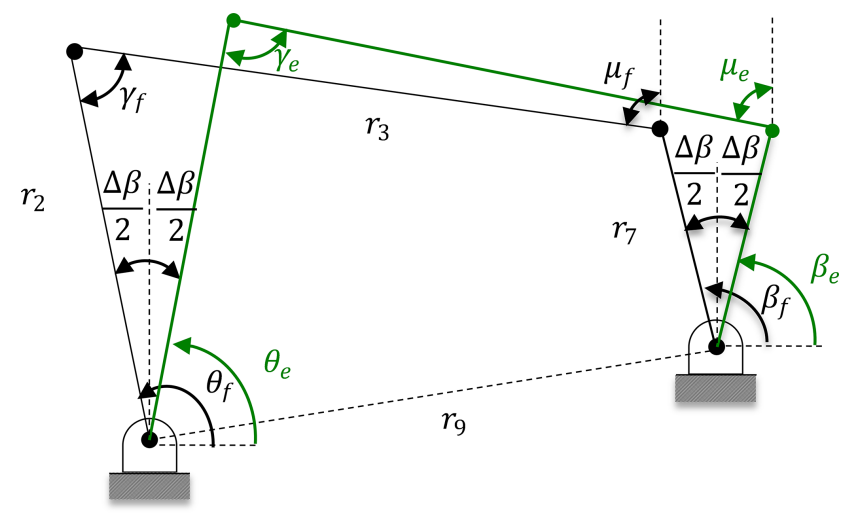

Figure 6. Four-bar mechanism in forward and fully withdrawn positions.

2011). However, it is verified that transmission characteristics of a compliant mechanism will be similar to those of its rigid body counterpart, if the output loading is large enough relative to the stiffness of compliant links (Tanık, 2011).

\section{Design approach of the rigid body equivalent of the compliant slider-crank mechanism}

In this section, the design approach targets three main objectives. First objective is the minimization of the relative link rotations while maximizing the translational motion of coupler of the second four-bar mechanism. Because relative rotations of the links determine the deflection amount of the hinges of the compliant slider-crank mechanism. The second objective is to equate the relative rotation of links from a reference position. Because, it is well known that, equality of deflection in both directions minimizes deflection peaks in a compliant mechanism design. The third objective is to equate all relative rotations of the links to a specific value, if possible. By this way, all hinges of compliant slider-crank mechanism can be designed with same dimensions that yields a robust design. Generally, for compliant mechanisms, the dominant loading is due to bending. Bending stress is similar for the same hinges with same dimensions and deflections.

The design of the rigid body equivalence is performed considering these three objectives as follows: Initially, the first four-bar mechanism $\left(A_{0} A B E\right)$ is designed. This mechanism with input and output oscillations of $\Delta \beta$ (to obtain the same deflections) is synthesized by function generation for three precision points. The forward and fully withdrawn positions of the mechanism are presented in Fig. 6. Subscript $f$ is used for the fully withdrawn positon that is represented with black lines, whereas subscript $e$ is used for the forward positon that is represented by green lines in Fig. 6. Let the vertical position of links 2 and 7 corresponds to the undeflected position of the related flexural hinges. Also let the forward and fully withdrawn positions are achieved when links 2 and 7 move at an angle of $\Delta \beta / 2$ from the vertical position.

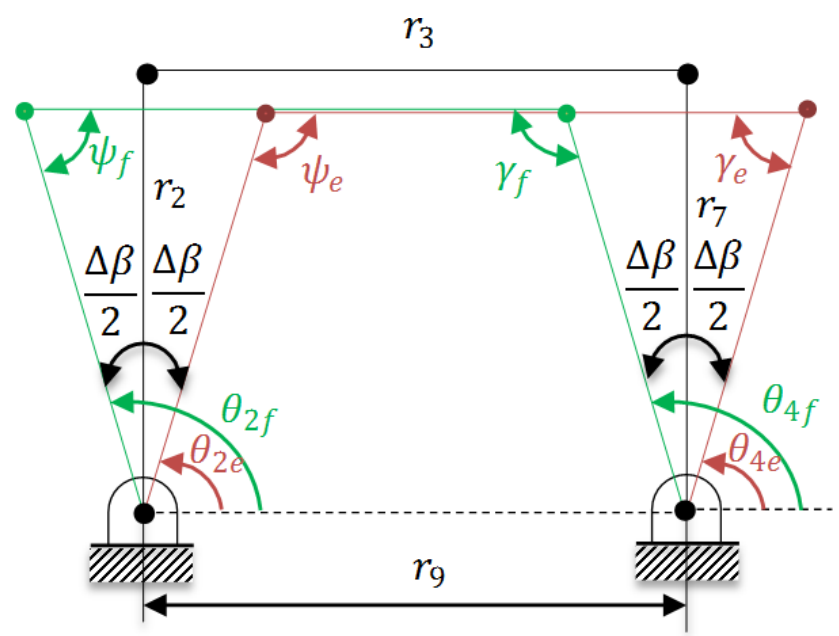

Figure 7. Four-bar mechanism in three positions.

A parallelogram four bar mechanism as presented in Fig. 7 is a neat solution according to the requirements. Green, black, and red lines represent the fully withdrawn, undeflected, and forward positions of the mechanism respectively. With this approach, the angle between the extreme positions of links 2 and 7 is $\Delta \beta$, and the undeflected position is exactly in the middle of these two positions. In other words, the extreme positions are symmetric with respect to the undeflected (vertical) position.

Considering the free design parameters, an infinite set of solution is available. However, in every solution it is determined that $r_{2}$ and $r_{7}$ are equal to each other that yields parallelogram four-bar mechanism. After function generation synthesis for three precision points, we obtained a solution where the complete mechanism is formed from two cascade parallelogram four-bar mechanisms. In Fig. 8 this mechanism is sketched in three positions that are undeflected, forward, and fully withdrawn positions. Remind that, $r_{7}$ is equal to $r_{5}$ that is obtained from the imaginary parallelogram fourbar mechanism. $L$ can be chosen as a free design parameter as in Eq. (6).

$r_{2}=r_{5}=r_{7}=L$

Links 2, 3, 7, and 9 forms a parallelogram four-bar, therefore all of the compliant segments perform the same angular displacement between the forward and fully withdrawn positions of the mechanism as in Eq. (7).

$$
\left(\psi_{e}-\psi_{f}\right)=\left(\gamma_{f}-\gamma_{e}\right)=\left(\theta_{2 f}-\theta_{2 e}\right)=\left(\theta_{4 f}-\theta_{4 e}\right)
$$

Then, from parallelogram four-bar mechanism, the equality of $r_{3}$ and $r_{9}$ can be written as:

$r_{3}=r_{9}$ 


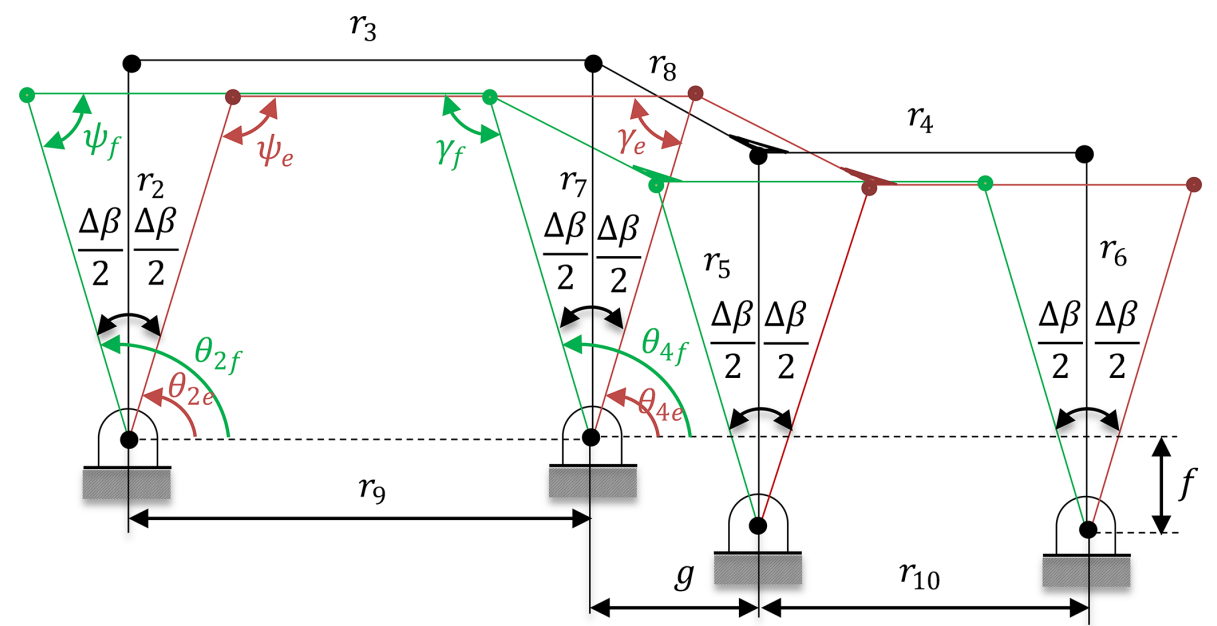

Figure 8. Four-bar mechanism in three positions (forward-undeflected-fully withdrawn) with design parameters.

Kinematic analysis of the mechanism is simple due to the symmetrical link proportions: The stroke of the mechanism which is the horizontal motion of $r_{4}$ is:

$$
\begin{aligned}
& \Delta S_{\text {total }}=2 L \sin (\Delta \beta / 2) \\
& \Delta S_{\text {left }}=\Delta S_{\text {right }}=L \sin (\Delta \beta / 2) .
\end{aligned}
$$

The axis drift of $r_{4}$ can be defined as:

$\Delta e=\frac{L}{2}(1-\cos (\Delta \beta / 2))$.

\section{Design of the fully compliant slider-crank mechanism}

Generally, if deflections of flexural hinges are large, stresses of flexural hinges are also high. Thus, in practice, deflection values should be kept in a feasible range. By using the rigid body replacement method, the fully compliant slider-crank mechanism can be dimensioned as shown in Fig. 9 .

As shown in the PRBM, links 4-6 form a parallelogram four-bar mechanism where link 5 and 6 correspond to fixed guided beams. According to the length of the top and bottom parts of long compliant segments are equal to $\frac{(1-\gamma) m}{2}$ and length of the middle part is equal to $\gamma m$ which is also equal to $r_{6}$; where $\gamma$ is characteristic radius factor and it can be determined from reference (Howell, 2001) as: $\gamma=0.852$ for $(\Delta \beta / 2)_{\max }=65^{\circ}$. Rigid segments 3 and 4 are aligned. Thus, Eqs. (11) and (12) can be determined referring to Fig. 9 as:

$h+\frac{(1-\gamma)}{2} \frac{r_{6}}{\gamma}+r_{6}-f=r_{6}$

where $h$ is half thickness of upper rigid segment.

$f=h+\left(\frac{1-\gamma}{2 \gamma}\right) r_{6}$
Note that, $g, r_{3}$, and $r_{4}$ are the free structure parameters. The value of the characteristic radius factor $\gamma=0.8517$ for $n=0$ and $\phi=90^{\circ}$, with a parameterization limit of $\Theta_{\max }=64.3^{\circ}$ for the flexible beam with a constant end angle and parametric angle coefficient $c_{\theta}=0$ (Howell, 2001). The moment, $M_{0}$, that is required to maintain a constant end angle can be determined as:

$M_{0}=\frac{P m}{2}[1-\gamma(1-\cos \Theta)]$.

Maximum stress occurs at both ends of the beam, where the maximum moment occurs and has a value of:

$\sigma_{\max }=\frac{P a c}{2 I_{1}}$

where, $c$ is the distance from the neutral axis to the outer surface of the beam. The torsional spring constants, $K_{1}$ and $K_{2}$ for the springs are given in Eq. (15) as:

$K_{1}=\frac{E I_{1}}{l}, K_{2}=2 \gamma K_{\theta} \frac{E I_{2}}{m}$.

Maximum stress at complaint segments (Howell, 2001) $C C_{0}$ and $D D_{0}$ is:

$\sigma_{\max }=\frac{K_{\Theta} E \Theta t_{\mathrm{FG}} \gamma[1-\gamma(1-\cos \Theta)]}{L \sin (\phi-\Theta)}$.

The summary of the design procedure of the fully compliant slider-crank mechanism is presented in Table 1.

By using Table 1, numerous fully compliant slider crank mechanisms can be designed for different dimensions. This generalized design table will be beneficial during preliminary design stage satisfying very large slider strokes with acceptable stresses. 


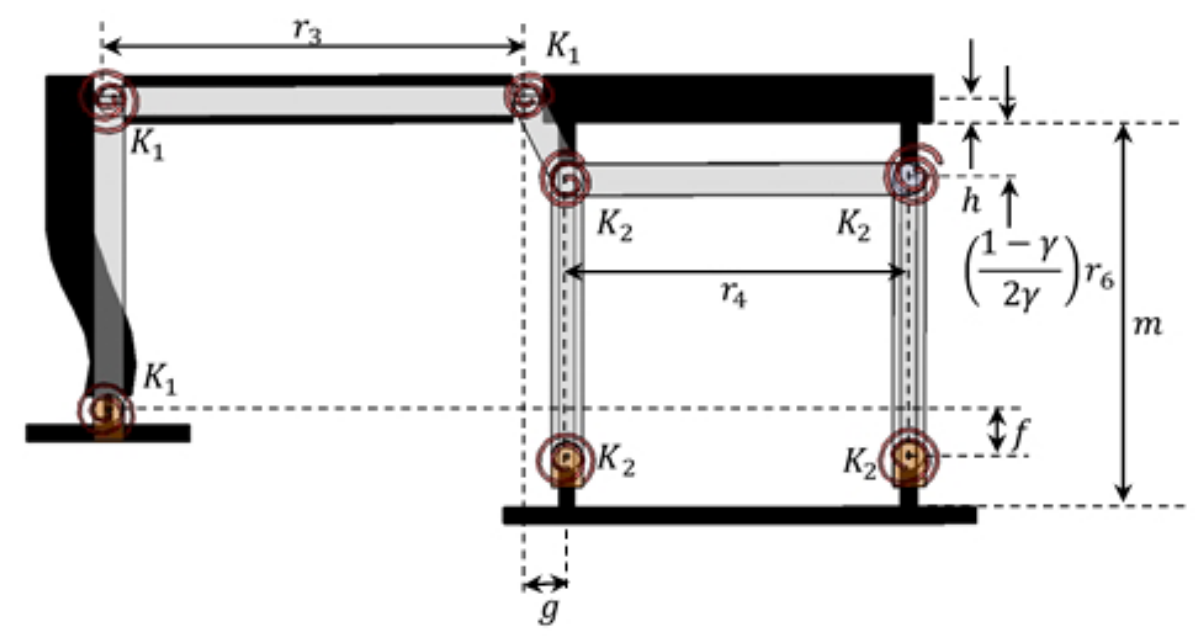

Figure 9. Fully compliant slider-crank mechanism overlapped with its PRBM.

Table 1. Design table of the fully compliant slider-crank mechanisms.

\begin{tabular}{llc}
\hline Stroke; & $\Delta S=2 L \sin \left(\frac{\Delta \beta}{2}\right)$ & Unit length \\
\hline Stroke in terms of size (compactness); & $\frac{\Delta S}{L}=2 \sin \left(\frac{\Delta \beta}{2}\right)$ & Unitless \\
\hline Stroke in terms of deflection; & $\frac{\Delta S}{L \beta}=2 \sin \left(\frac{\Delta \beta}{2}\right)$ & Unitless \\
\hline Axis drift in terms of deflection; & $\Delta e=\frac{L}{2}\left(1-\cos \left(\frac{\Delta \beta}{2}\right)\right)$ & Unit length \\
\hline Axis drift in terms of size; & $\frac{\Delta e}{L}=\frac{1}{2}\left(1-\cos \left(\frac{\Delta \beta}{2}\right)\right)$ & Unitless \\
\hline Maximum stress in Fixed Guided Beam; & $\sigma_{\max }=\frac{K_{\Theta} E \Theta t_{\mathrm{FG}} \gamma[1-\gamma(1-\cos \Theta)]}{L \sin (\phi-\Theta)}$ & \\
\hline
\end{tabular}

\section{A design example}

A fully compliant slider-crank mechanism is designed by using the method given in Sect. 6 and the equations in Table 1 as follows: let lengths of rigid segments 2,4 , and 6 be $L=100 \mathrm{~mm}$ and $\Delta \beta=40^{\circ}$. Material of the mechanism is selected as polypropylene which has modulus of elasticity $E=1.5 \mathrm{GPa}$ and yield strength of $40 \mathrm{MPa}$. The fixed guided beams are dimensioned as: thickness; $t_{\mathrm{FG}}=2.85 \mathrm{~mm}$, width; $w=15 \mathrm{~mm}$ and length; $m=117.4 \mathrm{~mm}$, since $L=100 \mathrm{~mm}$ and $\gamma=0.8517$. The stroke and axis drift of the slider are calculated analytically for $\Delta \beta / 2$ from Eqs. (9) and (10). Stresses at the fixed guided beam are determined analytically from Eq. (16) as:

$$
\begin{aligned}
& \Delta S=200 \sin \left(20^{\circ}\right)=68.4 \mathrm{~mm} \\
& \Delta e=\frac{100}{2}\left(1-\cos \left(20^{\circ}\right)\right)=3.02 \mathrm{~mm} \\
& \sigma_{\max }=\frac{2.68 \times 1500 \mathrm{MPa} \times 0.35 \mathrm{rad} \times 2.85 \mathrm{~mm} \times 0.85 \times\left[1-0.85\left(1-\cos 20^{\circ}\right)\right]}{100 \mathrm{~mm} \times \sin \left(90-20^{\circ}\right)}=34.4 \mathrm{MPa} .
\end{aligned}
$$

After the analytical design stage, by using rigid body replacement technique, a solid model of the mechanism is con- structed. Then FEA method is employed to compare output stroke, axis drift of the slider, and resultant stresses at the flexural hinges. The fully compliant slider-crank mechanism is analyzed for different sets of input rotations $(5,10,15$, $20^{\circ}$ ). For large rotations nonlinear analysis is selected. The resultant output stroke of the slider is presented in Fig. 10.

It is verified that, average of the output stroke and axis drift values of the slider are in close agreement with the analytical results. Resultant von-Mises stresses at the flexural hinges when the input rotation is set to its maximum value $\left(20^{\circ}\right)$ are presented in Fig. 11. The analytical maximum stress value at compliant segments is determined as $34.4 \mathrm{MPa}$ from Eq. (16), 32.9 MPa from FEA. The maximum stress value for the $20^{\circ}$ input is smaller than the yield strength.

A prototype of the mechanism is built for collecting experimental data. The mechanism is manufactured in one piece from polypropylene with a plate thickness of $15 \mathrm{~mm}$. During the manufacturing process 3 axis $\mathrm{CNC}$ router is used setting $7.5 \mathrm{~mm} \mathrm{~s}^{-1}$ cutting rate, $1.5 \mathrm{~mm}$ depth of cut and $4500 \mathrm{rpm}$ spindle speed. An experiment setup is established in order to measure the stroke and axis drift of the slider precisely as 


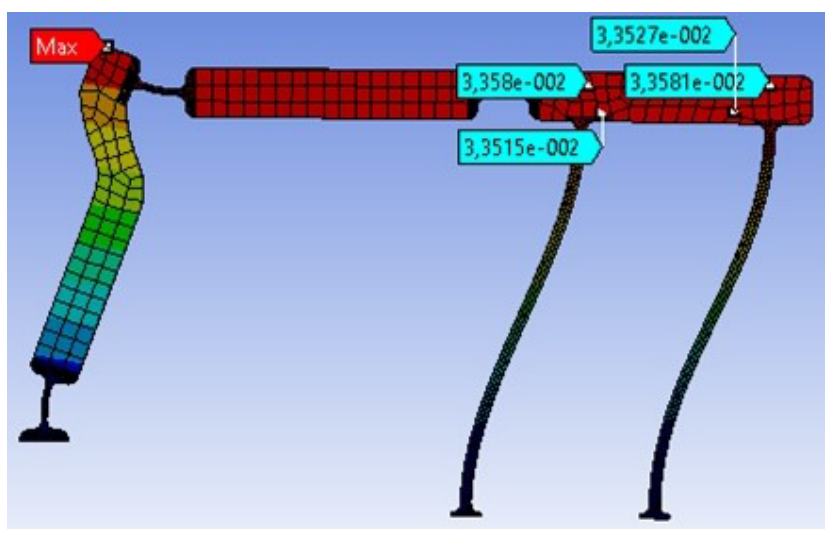

Figure 10. Stroke analyses for fully compliant slider-crank mechanism.

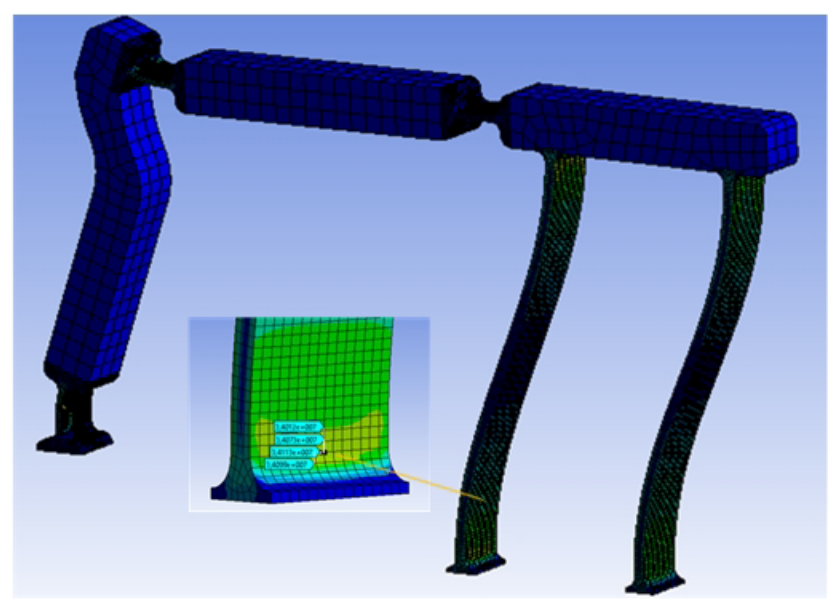

Figure 11. Maximum stress values at compliant segments for fully compliant slider-crank mechanism.

shown in Fig. 12. We compared the theoretical and experimental values as shown in Fig. 13. Note that position of the input is measured by a digital protractor and the output is measured by comparators.

The datum point (zero axis drift position) is selected as follows: the datum axis is the mid-point of highest and lowest positions of the output. Note that, there are differences in error values for the left and right axis drifts. Because, fine manufacturing of the long compliant segments with classical machining process is very hard. The manufacturing error that deteriorates initial parallelism of the long compliant segments causes difference between theoretical and experimental results. If the prototype were produced by plastic injection molding with a metal mold as in a mass production case, we would be able to achieve smaller error values.

It is calculated that the mean absolute error of all data points for the stroke measurements is $0.13 \mathrm{~mm}$. If this value is compared with the reference dimension $L=100 \mathrm{~mm}$, the error is $0.13 \%$. If this value is compared with stroke of

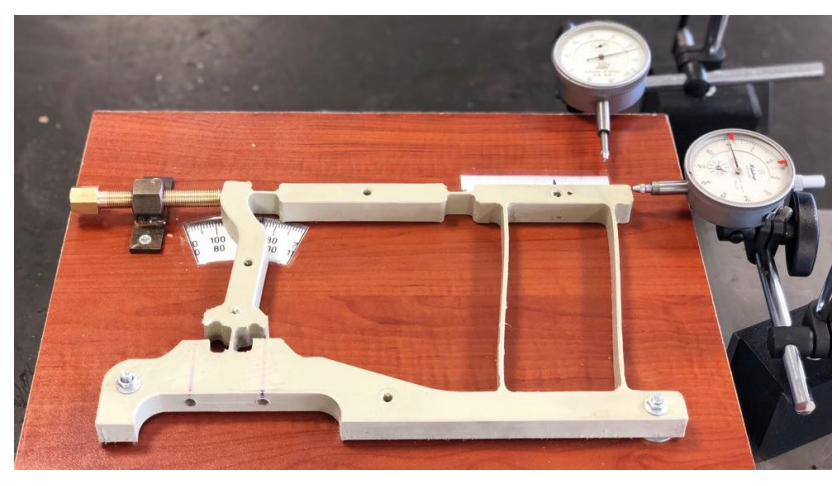

Figure 12. Experimental setup.

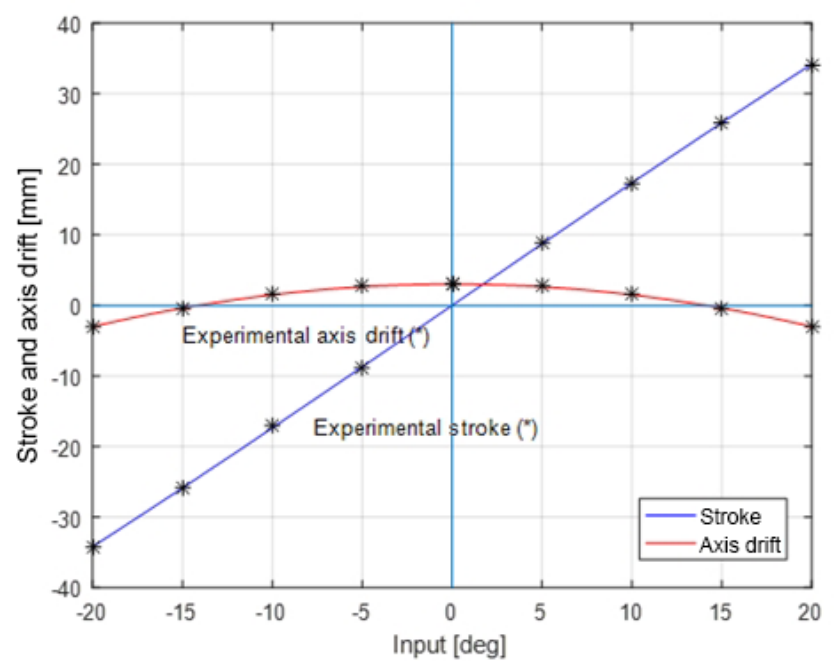

Figure 13. Theoretical and experimental stroke and axis drift of the fully compliant slider-crank mechanism.

the slider $\Delta S=68.4 \mathrm{~mm}$, the error is $0.19 \%$. For the axis drift the mean absolute error of all data points is $0.09 \mathrm{~mm}$. If this value is compared with the reference dimension $L=$ $100 \mathrm{~mm}$, the error is $0.09 \%$. If this value is compared with the stroke $\Delta S=68.4 \mathrm{~mm}$, the error is $0.13 \%$.

In the design of compliant mechanisms, there are always some errors due to roughness of PRBM approach. However, PRBM is not used for final dimensioning of a compliant mechanism. It is a tool which is used during the preliminary design stage. Precise dimensioning of compliant mechanism is generally finalized with finite element analysis tools. The design approach in this study is no exception to this issue.

\section{Discussion and conclusion}

Slider-crank is one of the most commonly used mechanisms in the industry and has numerous applications. In the literature, studies on "partially" compliant slider-crank mechanism can be found where the slider is a rigid joint. There are some fully compliant slider-crank mechanisms available 
however those are not optimized for large stroke and minimum link rotation. In this paper, a novel design procedure for a fully compliant slider-crank mechanism is proposed. In this design, the prismatic joint is replaced with a compliant parallel-guiding mechanism. The compliant parallelguiding mechanism is the output of the system that performs curvilinear translation provided that the output is not loaded with large forces. The novelty of this design approach is the slider's large stroke capacity. It is achieved by maximizing the translational motion of the slider while minimizing the relative link rotations thus stresses in compliant links.

A design approach for optimum link proportions of the PRBM of the fully compliant slider-crank mechanism is proposed. Input-output motion relationship of the mechanism and analytical stress values at flexural hinges are determined. In order to generalize the design approach, a procedure is employed and finally a design table that can be used for various tasks is presented. This table will be very beneficial for compliant slider crank designers.

As an example, a mechanism is synthesized using the design table. This mechanism is analyzed with FEA method to verify analytical results. It is shown that the results of the proposed theoretical model and FEA model are consistent. Finally, a single piece prototype is manufactured from polypropylene; thus, it has the advantage of ease of manufacturing when compared with the partially compliant mechanisms. Experimental setup is employed for stroke and axis drift measurements. It is observed that the output displacement and axis drift in the mathematical and the prototype are almost the same. Thus, it is verified that the proposed methods are consistent.

As a result, validity of the design approach is ensured by two ways. It is remarkable that the maximum error between the theoretical model and prototype is less than $0.2 \%$ (for slider stroke). This is a negligible error for a mechanism designed via PRBM approach where generally noticeable errors take place.

We believe this fully compliant slider-crank mechanism design procedure will be beneficial for many designers and may find many applications especially where backlash free design is required.

Data availability. Data can be made available upon reasonable request. Please contact Engin Tanık (etanik@ hacettepe.edu.tr).

Video supplement. The video file of the prototype is available at https://www.youtube.com/watch? $\mathrm{v}=$ wtjVkuwpUMg (last access: 6 February 2020).

Author contributions. ET proposed the original design and defined the methodology. ÇMT applied the methodology, performed analytical solutions, employed FEA, and wrote the paper. VP studied on the theoretical model. YY supervised ÇMT during the study.

Competing interests. The authors declare that they have no conflict of interest.

Review statement. This paper was edited by Lionel Birglen and reviewed by three anonymous referees.

\section{References}

Alqasimi, A., Lusk, C., and Chimento, J.: Design of a linear bistable compliant crank-slider mechanism, ASME J. Mech. Robot., 8, https://doi.org/10.1115/1.4032509, 2016.

Dao, T. P., and Huang, S. C.: Design and Analysis of Flexible Slider Crank Mechanism, Int. J. Aerosp. Mech. Eng., 8, 836843, https://doi.org/10.5281/zenodo.1337163, 2014.

Erkaya, S., Doğan, E., and Şefkatlıŏlu, E.: Analysis of the joint clearence effects on a compliant spatial mechanism, Mech Mach. Theory, 104, 255-273, 2016.

Hao, G., Li, H., Nayak, A., and Caro, S.: Design of a Compliant gripper with Multi-mode Jaws, J. Mech. Robot., 10, 031005 , https://doi.org/10.1115/1.4039498, 2018.

Hongzhe, Z., Shusheng, B., and Jingjun, Y.: A novel compliant linear-motion mechanism based on parasitic motion compensation, Mech. Mach. Theory, 50, 15-28, 2012.

Howell, L. L.: Compliant Mechanisms, John Wiley \& Sons, Inc., New York, USA, 2001.

Howell, L. L. and Midha, A.: A loop-closure theory for the analysis and synthesis of compliant mechnasims, ASME J. Mech. Design, 118, 121-125, 1996.

Li, X. C., Li, Y., Ding, B. X., and Xu, H. Y.: An investigation on kinematics and dynamics performance of a novel 3-PRCcompliant parallel micromanipulator, Adv. Mech. Eng., 10, 1-9, https://doi.org/10.1177/1687814018789800, 2018.

Liu, P. and Yan, P.: A modified pseudo-rigid-body modeling approach for compliant mechanisms with fixed-guided beam flexures, Mech. Sci., 8, 359-368, https://doi.org/10.5194/ms-8-3592017, 2017.

Pardeshi, S., Kandharkar, S., and Deshmukh, B.: Monolithic compliant slider crank mechanism for motion, Proc. Mater. Today, 4 1677-1682, https://doi.org/10.1016/j.matpr.2017.02.007, 2017.

Parlaktaş, V. and Tanık, E.: Single piece compliant spatial slidercrank mechanism, Mech. Mach. Theory, 81, 1-10, 2014.

Pavlović, N. T. and Pavlović, N. D.: Compliant mechanism design for realizing of axial link translation, Mech. Mach. Theory, 44, 1082-1091, 2009.

Shigley, J. E. and Uicker, J. J.: Theory of Machines and Mechanisms, McGraw-Hill Book Co., New York, USA, 1980.

Tanık, E.: Transmission angle in compliant slider-crank mechanism, Mech. Mach. Theory, 46, 1623-1632, 2011.

Trease, B. P., Moon, Y., and Kota, S.: Design of large displacement compliant joints, ASME J. Mech. Design, 127, 788-798, 2005.

Yang, T. S., Shin, P. J., and Lee, L. L.: Design of a spatial compliant translational joint, Mech. Mach. Theory, 94-114, 2016. 
Yu, Y. Q., Zhu, S. K., Xu, Q. P., and Zhou, P.: A novel model of large deflection beams with combined end loads in compliant mechanisms, Precis. Eng., 43, 395-405, https://doi.org/10.1016/j.precisioneng.2015.09.003, 2016.
Zhao, H., Han, Z., Zhang, L., and Shusheng, B.: Design of a stiffness-adjustable compliant linear-motion mechanism, Precis. Eng., 48, 305-314, 2017. 